\title{
Prediction of Tonal Underwater Noise Pattern from Cavitating Propellers with Special Attention to Ice Cover Effects
}

Heinrich Streckwall, Ph.D.

Herbert Bretschneider,

Hamburg Ship Model Basin

\begin{abstract}
To predict underwater noise spectra associated to regular occurrence of propeller cavitation we have extended an existing method [1] (used for the prediction of fluctuating hull pressures) to become applicable for effects that are linked to a finite speed of sound. In [2] an intermediate approach was realized where (besides the hull) far field boundaries were introduced but the incompressible flow assumption was kept. However compressibility effects become noticeable in the far field, which may be judged to start at some 2-3 propeller-diameters distance from the centre of the cavitation events, if we confine to emissions at $1^{\text {st }}-4^{\text {th }}$ blade frequency. It was a logical continuation of our former efforts to realize a compressible flow model and integrate the propeller as a noise source. Having increased the functionality of our approach by referencing the speed of sound, the precision of the method was also somehow reduced. In our former approach, like in comparable approaches (see for instance [3] and [4]), the singularity system generating the near field propeller induced pressures involved various sources and vortices distributed on the propeller blades. With our current compressible approach this complexity was dropped, as a single point source substitutes the cavitating propeller. Such a simplification correlates with the assumption, that the monopole character of a noise source is decisive for the far field noise levels. In this contribution we outline the steps characterizing the procedure for predicting tonal underwater noise from cavitating propellers. In the first step a Vortex Lattice Method (VLM) is used to access the cavitation pattern on the propeller with special focus on the cavity volume attached to one blade. The second step accumulates the distributed cavities to establish a fluctuating point source of equivalent far field noise characteristic. As relevant limits the hull, the free surface, the sea bottom and an ice cover are introduced. Using finally a Boundary Element Method (BEM) approach the relevant noise characteristics are derived, accounting for external boundaries and for the finite speed of sound. The results provided here are focused on a comparative treatment of different scenarios, mainly addressing ice cover effects at finite the water depth.
\end{abstract}

Keywords: Cavitation, underwater noise, ice-covered areas

\section{Modelling}

\section{Cavitation modelling}

The method which is invoked later to track the emitted noise can be considered a 'real' boundary element method i.e. the elements which make up the hull surface and other limiting areas are exactly at the boundaries, covering them without gaps. For the propeller with its relatively thin sections one may reference as well a simpler approach, namely a Vortex Lattice Method (VLM). Such a method is available and in permanent usage at the Hamburg Ship Model Basin (HSVA), called 'QCM'. For an application in the field of propeller noise emission the numerical load is on the cavitation model of QCM. Below we summarize the characteristics of this model:

QCM Cavitation model features

- Two-phase flow modeling: a) introducing (in the cavitation effected areas) added sources to simulate cavity displacement and added vortices to deriveoverall changes in camber, b) starting cavities at the blade leading edge and c) allowing for 'blunt' termination at the cavity end (no closure, i.e. no balance of added sources and sinks requested).

- $\quad$ Coupling with unsteady flow analysis: 'Fully wetted' blade surface pressure provided unsteady to feed steady cavitation model in each time step (quasi-steady approach). 
- $\quad$ Numerical scheme: 2D cavitation model solving for cavitation extent strip-wise on 3D blade surface, singularities kept fixed on mean surface.

- Cavitation types addressed: sheet cavitation on blade suction side and blade pressure side, supercavitation on suction side.

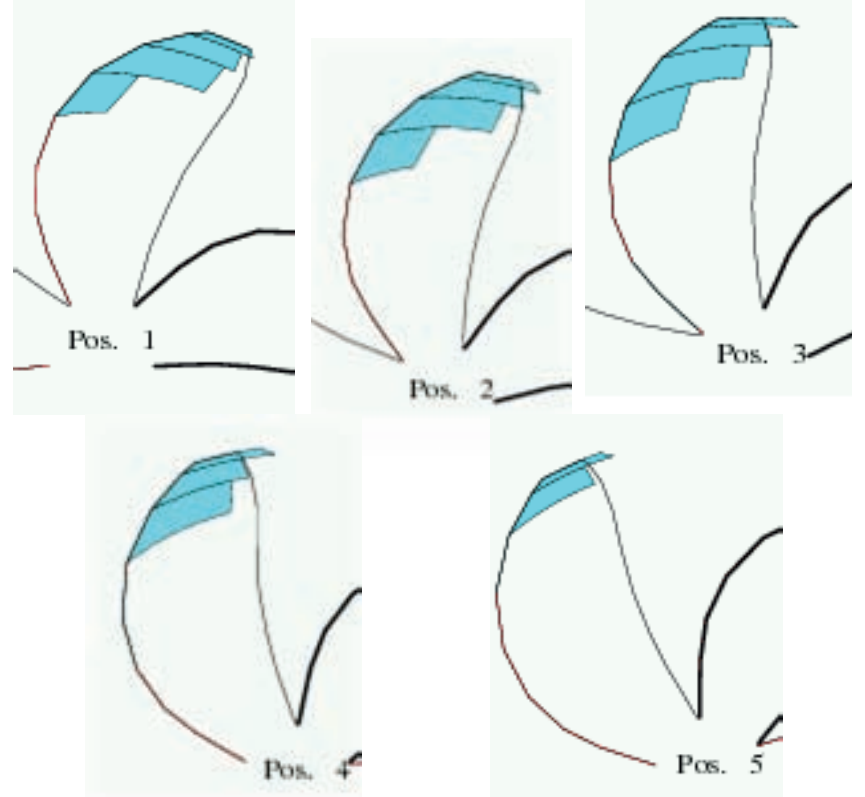

Fig. 1. Typical cavity pattern from numerical analysis (here VLM method QCM was used)

The fundamental information required from the propeller method to feed the 'noise tracking' procedure is given by the time function of the cavity volume. This information is linked to the added sources, which simulate the displacement effect of the cavity.

Provided a wake field is available (from measurements or calculations) and the propeller geometry was converted into a VLM-geometry the process to calculate the cavity volume can be started. The cavitation extent in Figure 1 represents a typical result of the VLM approach. As mentioned above, the vapor filled areas on the blade surface are linked to additional sources within the Vortex Lattice.

From the section wise cavity thickness, available via the VLM approach, one can derive the time function of the cavity volume attached to one blade. Finally a summation over all blades gives the volume history valid for all blades. Examples for both time functions can be taken from Figure 2, were the volumes are normalized by $\mathrm{R}^{3}$ with $\mathrm{R}$ as propeller radius. From a harmonic analysis one may define cavity volume amplitudes $\mathrm{V}_{\mathrm{o}}{ }^{\mathrm{n}}$ via $\left(\alpha_{\mathrm{n}}\right.$ is introduced to keep the amplitudes real):

$$
V(t)=V_{o}^{o}+\sum_{n=1} V_{o}^{n} \mathrm{e}^{\left(\mathrm{i} \alpha_{\mathrm{n}}-\mathrm{i} \omega_{n} t\right)}
$$

In the above $\omega_{n}=2 \pi n Z v$, with $Z$ for the numer of blades, $v$ for the propeller shaft frequency and $\mathrm{n}$ an integer mutiple ( $\mathrm{n}=1$ for the blade frequency, giving a frequency $\mathrm{Z}$ times higher than the shaft frequency $v ; n=2,3, \ldots$ for higher orders). For the pressure field at points of typically one radius $\mathrm{R}$ (or larger) distance from the cavitation activities, we consider this discrete singularity to be as representative as any detailed resolution of the cavitation pattern.

The single point source replacing the cavitating propeller in the noise tracking method is located in the propeller plane at the $12^{\circ \circ}$ position with an $80 \% \mathrm{R}$ distance to the shaft centre (see also Figure 3 ). While a general length scale is given by the propeller radius $\mathrm{R}$, a useful time scale is related to $1 / \mathrm{v}$, respectively to $1 / \Omega=1 /(2 \pi \nu)$ with $\Omega$ the related angular frequency. From the volume function $V(t)$, a source strength $\mathrm{Q}(\mathrm{t})$ may be derived by time derivative. Non-imaginary source strength amplitudes $\mathrm{Q}_{\mathrm{o}}{ }^{\mathrm{n}}$ are then obtained to represent $\mathrm{Q}(\mathrm{t})$ as follows: $Q(t)=Q_{o}^{o}+\sum_{n=1} Q_{o}^{n} \mathrm{e}^{i\left(\alpha_{\mathrm{n}}-\omega_{n} t-\pi / 2\right)}$. Similar to the role of $\alpha_{n}$, which keeps the volume amplitude $V_{0}{ }^{n}$ real, the role of $-\pi / 2$ in the above is to keep the source amplitude $\mathrm{Q}_{\mathrm{o}}{ }^{\mathrm{n}}$ real. As $\mathrm{Q}_{\mathrm{o}}{ }^{\mathrm{n}}=\omega_{\mathrm{n}} \mathrm{V}_{\mathrm{o}}{ }^{\mathrm{n}}$ and $\Omega=2 \pi v$, the normalized cavity volume amplitudes $\mathrm{V}_{\mathrm{o}}^{\mathrm{n}} / \mathrm{R}^{3}$ give normalized source amplitudes

$$
\mathbf{Q}_{\mathrm{o}}{ }^{\mathrm{n}} / \mathbf{\Omega} \mathbf{R}^{3}=\mathbf{n Z V _ { \mathrm { o } }}{ }^{\mathrm{n}} / \mathbf{R}^{3}
$$

To represent a generic propeller, which could be an average bulker- or VLCC- propeller one can use typical normalized source strength amplitudes $Q_{-} \mathrm{o}^{\wedge} \mathrm{n}$ depending on the considered blade raten as given below:

$$
\left(Q_{\mathrm{o}}{ }^{\mathrm{n}}\right) /\left(\Omega \mathbf{R}^{3}\right)=\mathbf{0 . 0 0 0 8 5 / \mathrm { n }}(\mathrm{n}=1,2,3, \ldots)
$$

On the hull this will lead to roughly $1 \mathrm{kPa}$ in full scale at blade frequency (in case of typical full scale propeller dimensions and in case of a typical full scale shaft frequency). We used $\mathrm{D}=7.9 \mathrm{~m}$ and $v=1,66 \mathrm{~Hz}$ in the present case. The Sound Pressure Level $=\mathrm{SPL}=20^{\star} \log \_10\left(\mathrm{p}_{\mathrm{o}}{ }^{\mathrm{n}} / \mathrm{p}_{\mathrm{ref}}\right)$, with $\mathrm{p}_{\mathrm{ref}}=$ $=10^{-6} \mathrm{~Pa}$, introduces a logarithmic scale, which is usually invoked for tracking the pressure amplitudes. $\mathrm{p}_{\mathrm{o}}{ }^{\mathrm{n}}(\vec{r})$ is the pressure amplitude related to the $n$-th blade frequency registered at $(\vec{r})$ in the mono-frequent signals caused by the fluctuating source $\mathrm{Q}_{\mathrm{o}}{ }^{\mathrm{n}}$. The location $(\vec{r})$ may be taken at any point in the flow, e.g. at the hull and in the far field. Note, that for $\mathrm{p}_{\mathrm{o}}{ }^{\mathrm{n}}$ we insert the full pressure amplitude i.e. we do not base the sound pressure level on the root mean square of the harmonic time signal. In one of the figures below, we will as well apply the SPL definition for an instantaneous pressure pattern.

Addressing the repeatability of the cavitation process we assume, that any model experiment or any calculation approach may not provide reliable data to run our point source model for full scale predictions (neither for hull pressure amplitudes nor for noise emission) at frequencies higher than the 3 times the propeller blade rate.

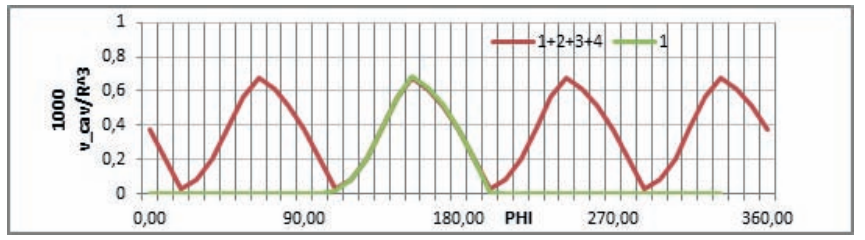

Fig. 2. Time function of normalized cavity volume valid for one blade (' 1 ') and, by superposition, for the whole 4-bladed propeller (' $1+2+3+4$ ') using the angular position (PHI) of a reference blade instead of time on the horizontal axis 


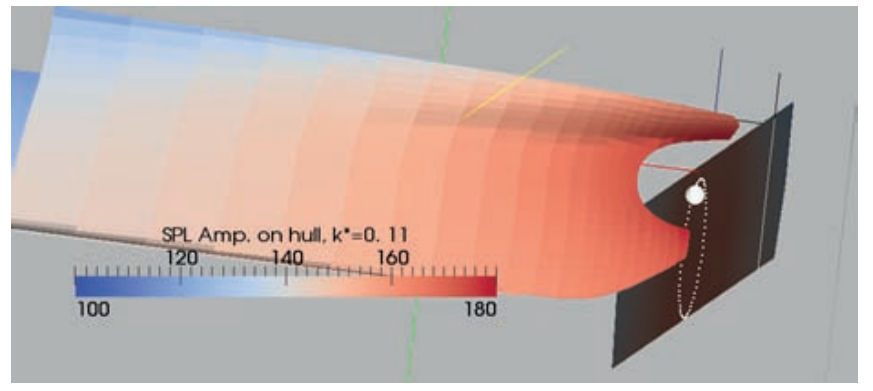

Fig. 3. The actual simulation is driven by a point source at $0.8 \mathrm{R}$ representing a 4-bladed propeller of $\mathrm{D}=7.9 \mathrm{~m}$ operating at $1.66 \mathrm{~Hz}$. Using the proposed formula (3) for the source strength, these settings comply with a max $1^{\text {st }}$ blade frequency hull surface pressure amplitude of $1.0[\mathrm{kPa}]$ corresponding to a max sound pressure level SPL of 180.0 (view from Port/below).

\section{Sound propagation modelling}

The single point sourcerepresenting the cavitating propeller is a rigorous modelling measure. A similar characteristic (rough but efficient) may be stated for the free surface model, which enters the calculation. It is assumed that negative mirror images of all singularities and boundaries acting under water are to be installed above the free surface. This shall ensure the free surface boundary condition, expressing that a free surface cannot withstand any other pressure than the surrounding air pressure. The link between the mirror system and the demand on the pressure at $\mathrm{z}=0$ (free surface location) will become obvious when we discuss in more detailthe sound propagation model. In general this acoustic model is based on a velocity potential $\phi(\vec{r}, \mathrm{t})$ representing the point source, all contributions from boundaries as well as all mirror images and linked to the velocity field via $\vec{v}(\vec{r}, \mathrm{t})=\operatorname{grad} \phi(\vec{r}, \mathrm{t})$.

In the compressible case a reasonable approximation for the continuity equation can be expressed as follows:

$$
\frac{\partial}{\partial t} \varrho^{\prime}+\varrho_{o} \operatorname{div} \vec{v}=0
$$

$Q$ ' stands for small changes of the density around the mean value $Q_{0}$. It relates to the disturbed pressure via the speed of sound $\mathrm{c}$ as given below:

$$
p^{\prime}(\vec{r}, \mathrm{t})=c^{2} \varrho^{\prime}(\vec{r}, \mathrm{t})
$$

According to the unsteady Bernoulli equation the pressure perturbations p' from equilibrium may be linked to the velocity potential according to:

$$
p^{\prime}(\vec{r}, t)=\varrho_{0} \dot{\phi}(\vec{r}, t)
$$

Here $\phi$ represents the first time derivative. Thus the continuity equation (4) can be summarized to a linear wave equation (below $\dot{\phi}$ for the second time derivative of $\phi$ and $\nabla^{2}$ for div grad ):

$$
\nabla^{2} \phi(\vec{r}, \mathrm{t})=1 / c^{2} \ddot{\phi}(\vec{r}, t)
$$

The time dependency is shifted to the frequency domain, so that a n-th blade frequency tonal emission from the point source at related $\omega_{\mathrm{n}}=2 \pi v \mathrm{nZ}$ (with strength $\mathrm{Q}_{\mathrm{o}}$ and location $\vec{r}_{Q \text { ) would cause an undisturbed potential }}$

$\phi_{\infty}^{n}(\vec{r}, t)=Q_{0}^{n} /\left(4 \pi\left|\vec{r}-\vec{r}_{Q}\right|\right) e^{i \omega_{n}\left(\left|\vec{r}-\vec{r}_{Q}\right| / c-t\right)}$

The wavenumber $\mathrm{k}_{\mathrm{n}}=\omega_{\mathrm{n}} / \mathrm{c}$ represents a key-parameter and should be suitably normalized. $\mathrm{k}_{\mathrm{n}}$ is made non-dimensional here as $\mathrm{k}_{\mathrm{n}}{ }^{*}=\left(\omega_{\mathrm{n}} / \mathrm{c}\right) \mathrm{R}$, so that $\mathrm{k}_{\mathrm{n}}{ }^{*}$ quite directly expresses the ratio of propeller radius $R$ to the sound wave length at $\omega_{n}$.

\section{Retardation}

An acoustic problem involving a periodically acting singularity and surrounding boundaries shows delays for the mutual interaction processes (the disturbance at $\vec{r}$ ' will need time to reach $\vec{r}$ ). For harmonic problems one may include delay effects via a phase offset which enters any interaction process. A specific delay time reads $\left|\vec{r}-\vec{r}^{\prime}\right| / \mathrm{c}$ and leads to a specific phase offset $\Delta \alpha=\left(\omega_{\mathrm{n}} / \mathrm{c}\right)\left|\vec{r}-\vec{r}^{\prime}\right|$. This procedure allows to rely basically on a former developed (and also potential based) 'near field approximation' addressing the pressure field caused by the propeller on the hull. It adds a mutual interaction factor

$$
\mathrm{e}^{\mathrm{i}\left(\omega / \mathrm{c}\left|\vec{r}-\vec{r}^{\prime}\right|-\omega t\right)}
$$

to any incompressible influence function holding between a boundary element at $\vec{r}^{\prime}$ and any point $\vec{r}$.

In the incompressible case we used dipole densities for the involved surfaces and boundaries to introduce a system of distributed singularities. The additional interaction factor from equation (9) gives a linear equation system in a similar manner as before. The unknown dipoles however now show a real and an imaginary part, doubling the number of equations and the size of the solution vector.

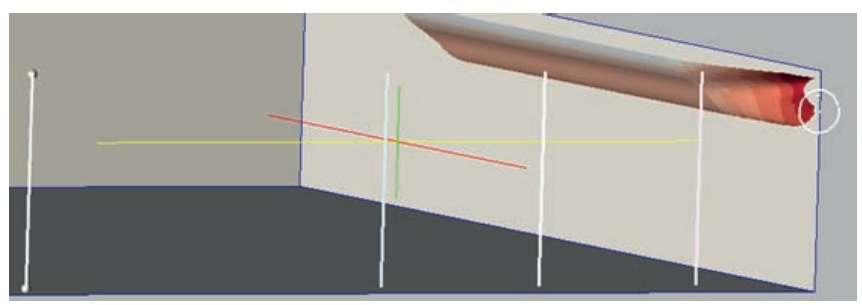

Fig. 4. This picture shows the hull, sea bottom, symmetry plane and vertical lines for probe locations. The probe coordinatesenterthe diagrams presenting the sound pressure levels (SPL) as function of sensor submergence (view from Port/behind). The vertical lines locate at the axial position of the propeller and in lateral distances of 6R, 14R, 22R and 40R from the mid ship plane. 


\section{Scenarios and results}

\section{Scenarios}

We were interested in a simulation that would give results similar to the outcome of a hypothetic experiment whereby a hydrophone is positioned at various depths and distances from the passing ship. We considered a normalized water depth of $10 \mathrm{R}$, which results to $39.5 \mathrm{~m}$ using $\mathrm{D}=7.9 \mathrm{~m}$. We were introducing a typical containership hull and a typical propeller tip clearance of $35 \% \mathrm{D}$. The propeller was assumed 4-bladed and $\mathrm{n}$ was set to $1.66[1 / \mathrm{s}]$ giving a normalized wave number $\mathrm{k} 1^{*}=(\omega \mathrm{n} / \mathrm{c}) \mathrm{R}=0.113$ for $\mathrm{n}=1$ (1st bade frequency). Besides $\mathrm{k} 1^{\star}$ we also did calculations with $\mathrm{k} 0^{*}=0$ and $\mathrm{k} 2^{*}=$ $0.226 . \mathrm{k} 0^{\star}=0$ simulates a sound source with extreme low frequency and enforces an amplitude pattern, which would hold in an incompressible medium at arbitrary frequency. $\mathrm{k} 2^{*}=0.226$ would correspond to the 2 nd blade frequency of the above mentioned 4-bladed propeller.

The three settings for the conditions at the upper liquid water limit were:

-free surface';

-ice channel' (ice starting on both sides at 10R from midship);

-rigid cover all-over'.

The normalized source strength was basically set as given in equation (3), however we introduced slight deviations from equation (3) to enforce the near field sound pressure levels to be the same for all conditions and frequencies. As a representative near field value we referenced the hull pressure amplitude directly above the propeller. This was enforced to read $1 \mathrm{kPa}$, independent of frequency and condition.

As already suggested the free surface condition was introduced by negative mirror images of the submerged source and boundaries. The ice channel condition includes a free surface part (the channel) and a rigid part (ice everywhere else). This ice scenario was modelled using negative mirror images again, however combining them with slightly submerged rigid panels representing the lower ice surface. The distributed singularities (dipole densities) on the ice panels became part of the solution. The model for the rigid cover (which may represent a situation similar to a ship model attached to the upper tunnel wall) involved positive mirror images of the submerged source and boundaries.

\section{Results}

The distribution of the pressure amplitudes may be traced in vertical direction from $\mathrm{z}=0$ down to the sea bottom as indicated by the vertical lines shown in Figure 4. We were selecting lines located at the axial position of the propeller in lateral distances of $6 \mathrm{R}, 14 \mathrm{R}, 22 \mathrm{R}$ and $40 \mathrm{R}$ from the mid ship plane. For the scenarios 'free surface', 'ice channel' and 'rigid cover all-over' the sound pressure levels may such be presented via xy-diagrams, whereby our vertical axis denotes the submergence and the horizontal axis stands for the SPL.
The plane covering the vertical lines at $6 \mathrm{R}, 14 \mathrm{R}, 22 \mathrm{R}$ and $40 \mathrm{R}$ was also referenced to provide contour plots of the sound pressure level.

To compare with results which would hold for the previous approach [1] we first considerd a point source at zero frequency, i.e. we started with a normalized wave number $\mathrm{kn}^{*}=(\omega \mathrm{n} / \mathrm{c}) \mathrm{R}=0.0$. As already noted, this would produce an amplitude pattern valid in an incompressible medium at any frequency. Figure 5 gives the contour plots of the sound pressure levels at $\mathrm{kn}^{\star}=0$. The ice channel scenario (Figure 5, mid) includes an indication of the ice-cover extent by a plate fixed on top.
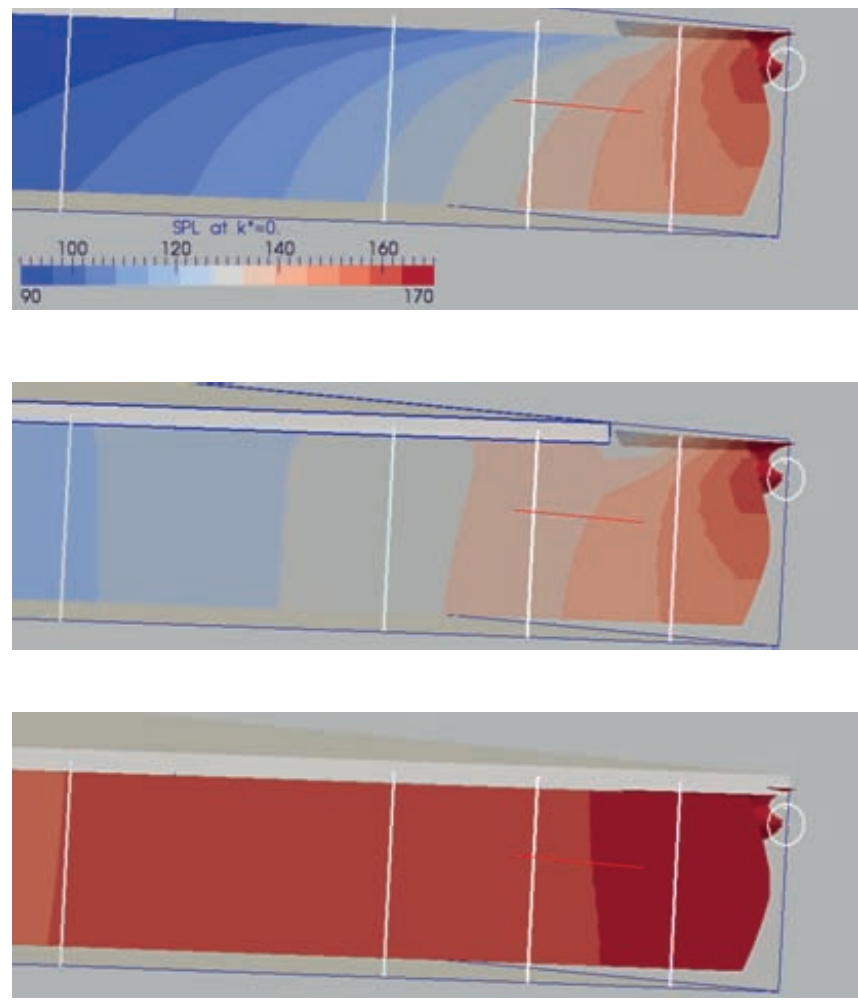

Fig. 5. Total SPL pattern in the $\mathrm{x}=0$ - plane (axial propeller position) for $\mathrm{k}_{\mathrm{n}}{ }^{*}=0$ case. Top: free surface scenario, middle: ice channel scenario, bottom: rigid surface scenario.

Next we treated a normalized wave number $\mathrm{k} \_\mathrm{n}^{\wedge *}=\left(\omega \_\mathrm{n} / \mathrm{c}\right)$ $\mathrm{R}=0.113$ which represents a typical 1st blade frequency $(\mathrm{n}=1)$ value for large ships. Figure 6 gives the contour plot of the sound pressure level in this case. Again the contour plot plane includes the vertical lines.

Finally we introduced $\mathrm{k} \_\mathrm{n}^{\wedge *}=\left(\omega \_\mathrm{n} / \mathrm{c}\right) \mathrm{R}=0.226$, i.e. we doubled the frequency of the point source to simulate the transmission of cavitation noise related to a 2 nd blade frequency $(n=2)$ typical for large ships. The results for this case can be taken from Figure 7 .

For an instant pressure picture we refer to Figure 8. Here we applied the sound pressure level definition to display the pressure pattern at a fixed time t_o. This instantaneous pressure field relates to the scenario 'rigid cover all-over, $\mathrm{k} \_\mathrm{n}^{\wedge *}=0.226^{\prime}$. 

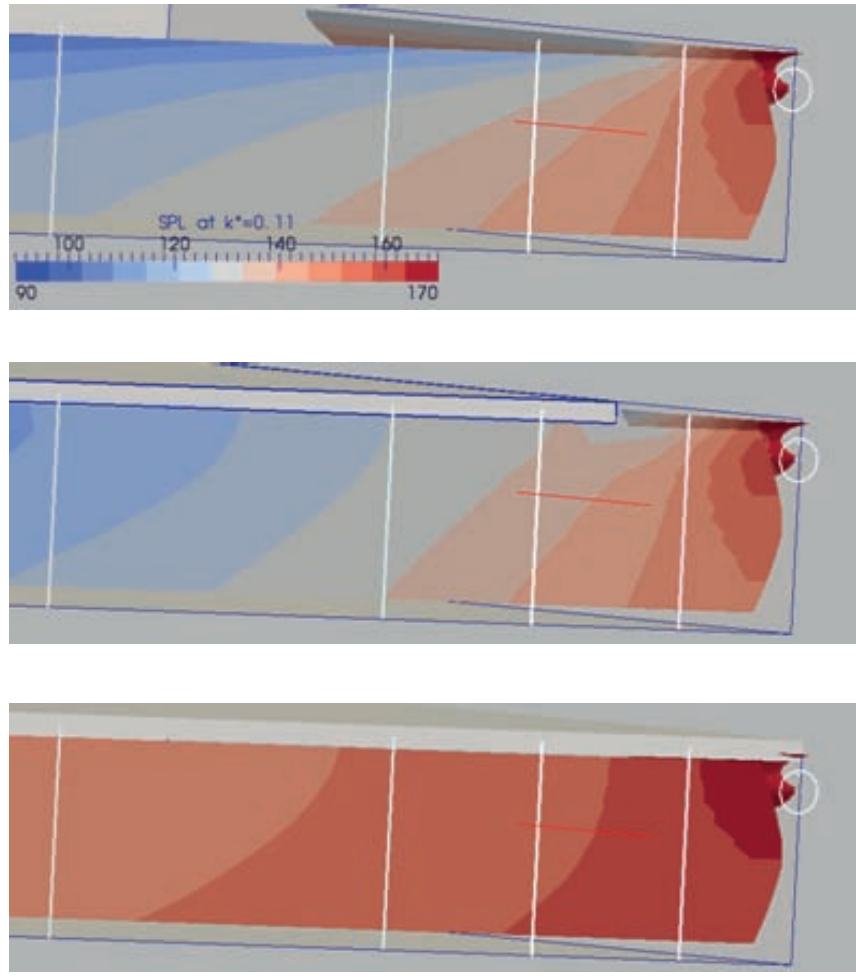

Fig. 6. Total SPL pattern in the $\mathrm{x}=0$ - plane (axial propeller position) $\mathrm{k}_{1}^{*}=0.113$ case. Top: free surface scenario, middle: ice channel scenario, bottom: rigid surface scenario.
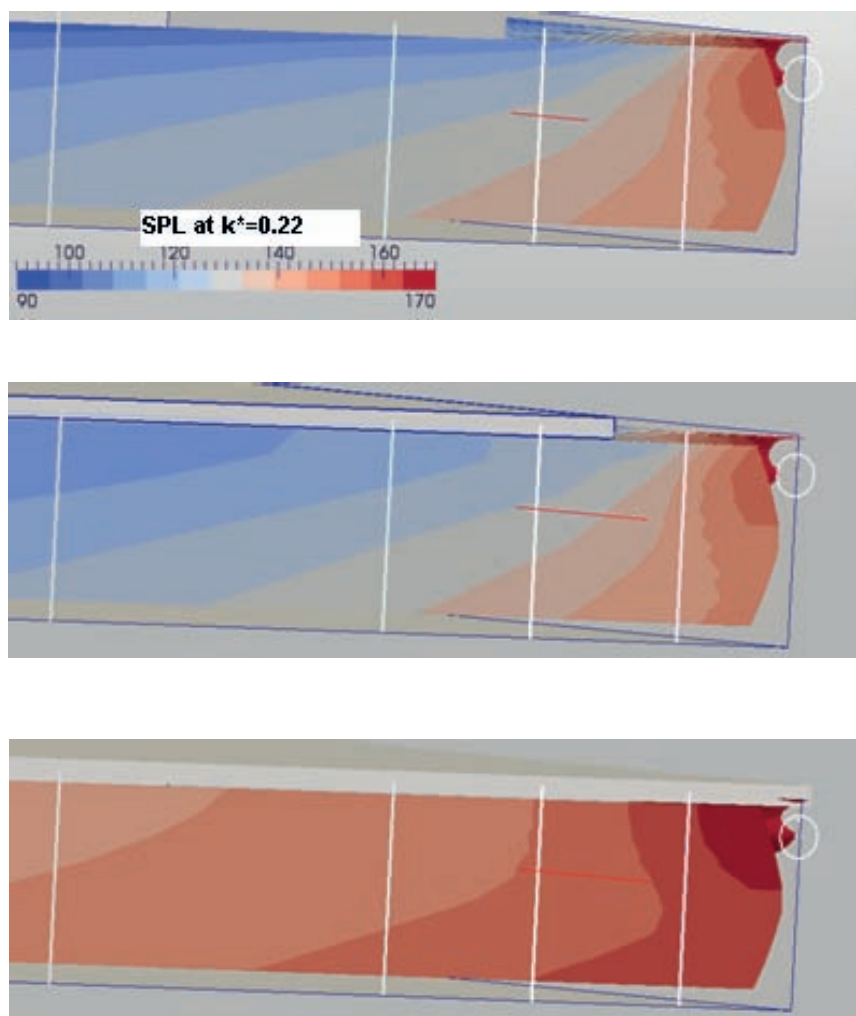

Fig. 7. $\mathrm{k} \_2^{\wedge *}=0.226(\mathrm{n}=2)$. Top: free surface, middle: ice channel, bottom: rigid cover all-over.

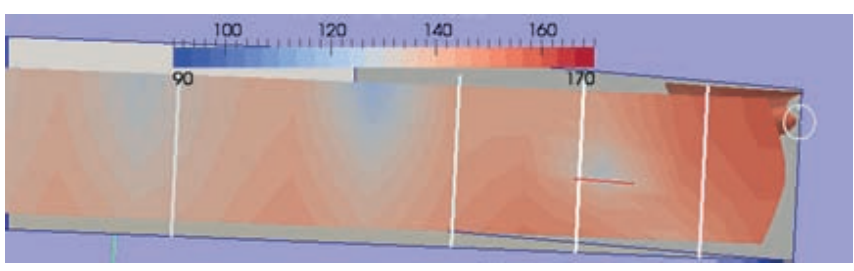

Fig. 8. Instant pressure field result (again presented as sound pressure level) related to the amplitude pattern given in Figure 7, bottom (rigid cover all-over, $\mathrm{k}_{\mathrm{n}}^{*}=0.22$ )
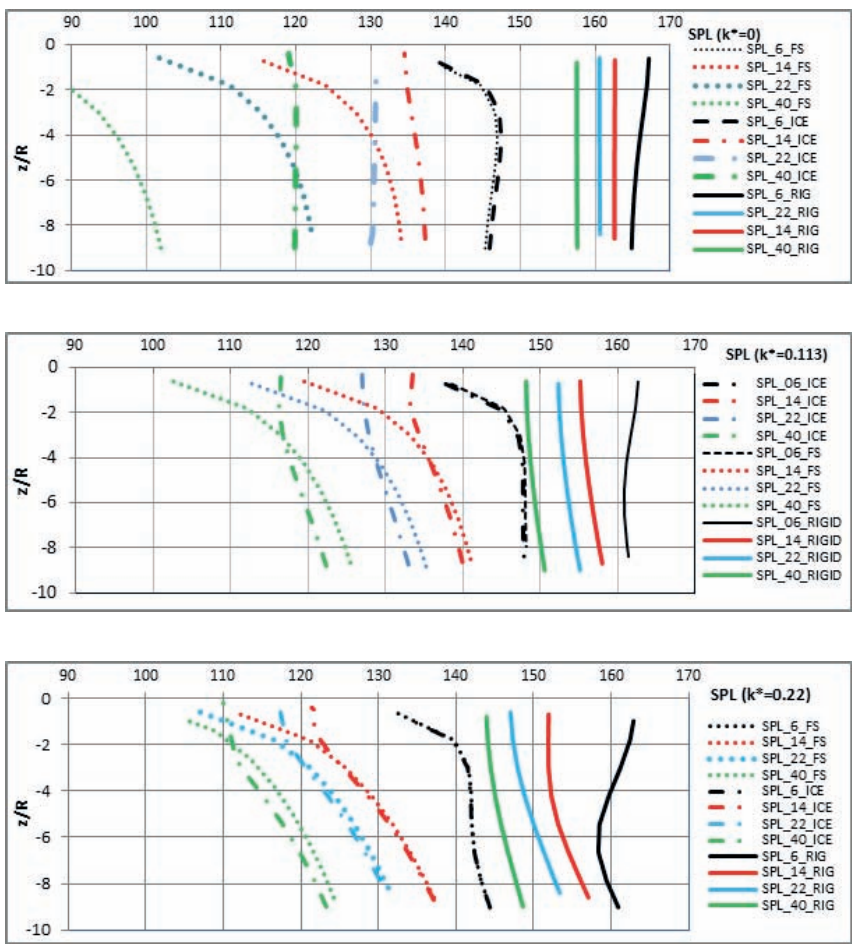

Fig. 9. The sound pressure level (SPL) runs along the horizontal axis while the submergence of sensors (positioned according to Figure 4 at $6 \mathrm{R}, 14 \mathrm{R}, 22 \mathrm{R}$ and $40 \mathrm{R}$ from the mid ship plane) runs vertically. The normalized wave numbers read $\mathrm{k}^{*}=0,0.113,0.226$. The conditions ' $\mathrm{RIG}$ ' (ridged cover), 'ICE' (ice channel) and 'FS' (free surface) refer the above mentioned scenarios.

Figure 9 shows the sound pressure level (SPL) obtained along the 4 vertical lines, when everything remains the same except for the scenario at $\mathrm{z}=0$ (upper limit for liquid water). Simulating with $\mathrm{k}_{\mathrm{n}}{ }^{*}=0$ an incompressible approach (the latter characterized by an amplitude result independent of the emission frequency) we arrive at the most extreme pattern as can be taken from the upper graph of Figure 9. It shows the strongest SPL-decay in lateral direction if the free surface scenario is invoked and exhibits the weakest lateral SPLdecrease if the rigid cover scenario is activated.

In Figure 9 the strong reduction of the SPL at the free surface is obvious for all emission frequencies $\mathrm{k}_{\mathrm{n}}{ }^{*}=0,0.113$ and 0.226 . Precisely at the free surface the SPL reads formally $-\infty$ corresponding to zero pressure amplitude. The SPL for the ice channel behaves very similar to the free surface case, as long as the ice edge is not reached. Away from this edge, 
i.e. under ice, the character of the curves (but not the levels) switches over to the ridged cover case. In any case we can hardly observe a decrease of the sound pressure level in the negative vertical direction, an effect surely related to our very limited water depth. If we step from $\mathrm{k}_{\mathrm{n}}{ }^{*}=0$ to finite emission frequencies, it appears to be just the other way round. At the highest frequency we register a marked increase of the SPL with rising distance from $\mathrm{z}=0$.

\section{Conclusion}

Using our approach to predict the low frequency noise signature of a propeller we concentrated on the influence of the far field boundaries. This exercise is including the scenario of a limited open water channel surrounded by ice. The way in which low frequency noise propagates under ice is of some interest when the risks of transportation and exploitation in the Arctic areas are studied. People are concerned about the effect of underwater noise on marine mammals.

Besides the settings at the upper liquid water limit we also varied the frequency of noise emission.

The water depth represents a parameter, which was not yet varied but fixed to a typical shallow water level in the current study.

We demonstrated that an incompressible approach delivers the most extreme results, meaning that it supplies the strongest decay of the sound pressure levels when the free surface scenario is invoked and the weakest decay when the rigid cover scenario is activated. The ice channel scenario turns out to be sensitive to the actual frequency of the sound source. Under the ice, in combination with shallow water, we could not observe a decline of the sound pressure level with increased submergence.

In view of the noise pollution under ice, we may emphasize to keep ice channels as broad and open as possible, especially in a shallow water situation. It may also be an option to equip blunt ships with special devices, like unconventional preducts. It has been demonstrated in [5], that such devices may lower the hull pressure amplitudes for the 2nd and higher blade frequencies. Accordingly the noise emitted at these frequencies would also be reduced.

\section{References}

1. Streckwall, H.: Numerical Models for Cavitationand Propeller Pressure Fluctuations. Jahrbuch der Schiffbautechnischen Gesellschaft, 97th Edition; pp 4449, Springer-Verlag Berlin and Heidelberg GmbH V, 2003

2. Streckwall, H., Bretschneider, H.: A Study on propeller noise emission with special attention to the influence of an ice coverage and finite water depth, 2nd Intern. Symp. on Marine Propulsors, SMP'11, Hamburg, Germany, 2011

3. Breslin, J.P., Van Houten, R.J., Kervin, J.E., Johnson, C.A.: Theoretical and experimental propeller-induced hull pressures arising from intermittent blade cavitation, loading and thickness, Soc. Nav. Archit. Mar. Eng. Trans. 90; 111-151, 1982

4. Kanemaru, T., Ando, J.: Numerical analysis of cavitating propeller and pressure fluctuation on ship stern using a simple surface panel method 'SQCM', SMP'11, Hamburg, Germany, 2011

5. Shin, H.-J., Lee, J.-S. ,Lee, K.-H., Han, M.-R.,Hur, E.-B.. Shin, S.-C: Numerical and experimental Investigation of conventional and un-conventional preswirl duct for VLCC', Int. Journ. of Naval Archit. and Ocean Engin., volume 5, issue 3, 2013, Pages 414-430

\section{CONTACT WITH THE AUTOR}

\author{
Heinrich Streckwall \\ Herbert Bretschneider \\ Hamburg Ship Model Basin, \\ Bramfelder Straße 164 \\ Hamburg, \\ GERMANY
}

